\title{
Evaluation of 5 Commercially Available Zika Virus Immunoassays
}

\section{David Safronetz, Angela Sloan, Derek R. Stein, Emelissa Mendoza, Nicole Barairo, Charlene Ranadheera, Leanne Scharikow, Kimberly Holloway, Alyssia Robinson, Maya Traykova-Andonova, Kai Makowski, Kristina Dimitrova, Elizabeth Giles, Joanne Hiebert, Rhonda Mogk, Sharla Beddome, Michael Drebot}

Because of the global spread of Zika virus, accurate and high-throughput diagnostic immunoassays are needed. We compared the sensitivity and specificity of 5 commercially available Zika virus serologic assays to the recommended protocol of Zika virus IgM-capture ELISA and plaque-reduction neutralization tests. Most commercial immunoassays showed low sensitivity, which can be increased.

$\mathrm{Z}$ ika virus is a mosquito-borne member of the family Flaviviridae, genus Flavivirus, that was originally discovered in 1947 in Uganda (1). For several decades, Zika virus seemed to be geographically restricted to equatorial Africa with a few documented incursions into Asia $(2,3)$. Although several studies demonstrated serologic evidence of human exposures to Zika virus across Africa, it was believed that this virus was not a major public health threat. However, in 2007, the epidemic potential of Zika virus became apparent when it was identified as the causative agent of an outbreak in Yap State, Federated States of Micronesia, which consisted of 49 confirmed cases, 59 probable infections, and dozens more suspected cases $(4,5)$. Since 2007, several epidemics have occurred across the Pacific Ocean Region, including an outbreak in 2013-14 with thousands of confirmed cases in French Polynesia (6).

In 2015, the first cases of Zika virus infection were confirmed in Brazil, which indicated the beginning of the largest outbreak recorded with autochthonous vectorborne transmission documented in $>65$ countries across the Americas $(2,3,7)$. Although it is still widely believed that most Zika virus infections in humans are asymptomatic or mild with self-limiting clinical manifestations, it is now documented that Zika virus infections can lead to major complications and long-term sequelae, including congenital birth Author affiliation: Public Health Agency of Canada, Winnipeg, Manitoba, Canada

DOI: https://doi.org/10.3201/eid2309.162043 defects, neurologic disorders, and prolonged risk for sexual transmission of this virus $(3,8)$.

Before 2007, only 14 laboratory-confirmed cases of Zika virus infection had been documented worldwide. Thus, it is not surprising that diagnostics for Zika virus were conducted only in specialized arbovirus reference laboratories (2). During the outbreak on Yap Island, samples were sent to the Arbovirus Diagnostic Laboratory, Centers for Disease Control and Prevention (Fort Collins, CO, USA), where molecular and serologic assays were quickly developed for confirmatory testing (5). Many of these in-house methods developed in 2007, including real-time molecular assays, an IgM-capture ELISA (MAC-ELISA), and a plaque reduction neutralization test (PRNT), have been used during the current outbreak. However, the magnitude of the outbreak, combined with the in-house production of key reagents involved in diagnostics of Zika virus infection, has taxed the few reference laboratories capable of producing, standardizing, and distributing such materials. Therefore, application and evaluation of sensitive and specific diagnostic assays, particularly those that can be used in frontline laboratories, has become a top public health priority.

Several laboratories and commercial vendors have developed and evaluated molecular assays for rapid identification of Zika virus RNA, and, in some instances, other clinically relevant arboviruses, such as dengue virus (DENV) and chikungunya virus, in acute-phase clinical specimens (9). However, high-throughput commercially produced immunoassays have proven to be more challenging because of strong serologic cross-reactivity of closely related flaviviruses, such as DENV. We compared the sensitivity and specificity of 5 commercially available Zika virus serologic assays to the recommended protocols of Zika virus MACELISA and PRNT.

\section{The Study}

Samples were submitted to the National Microbiology Laboratory of the Public Health Agency of Canada (Winnipeg, Manitoba, Canada) for arbovirus diagnostic testing. All samples were obtained from Canadian travelers who visited areas with known Zika virus transmission and consulted their physicians after symptoms consistent with Zika virus infection developed upon return.

We obtained deidentified samples from 75 patients. Thirty samples were from patients with serologically confirmed Zika virus infections; 10 from patients with 
confirmed Zika virus infections identified by 2-target real-time reverse transcription PCR (RT-PCR); 10 from patients with suspected Zika virus infections, which were subsequently identified as DENV infections; and 25 acutephase samples from flavivirus-negative persons tested by Zika virus RT-PCR and MAC-ELISA. Primary Zika virus diagnostic testing for all samples was conducted by using an in-house CDC-based MAC-ELISA and subsequent confirmation of Zika virus infection by cross-PRNTs for Zika virus and DENV, or molecular assays as described (5).

We evaluated 5 Zika virus immunoassays in this study. We tested a conventional IgM ELISA (EI 2668-9601 M; Euroimmun AG, Luebeck, Germany) and 3 MAC-ELISAs: Zika Virus Detect (InBios International Inc., Seattle, WA, USA); Ab213327 (Abcam, Cambridge, UK); and NovaLisa ZVM0790 (Novatec Inc., Baltimore, MD, USA). On the basis of preliminary testing, we also tested the Euroimmun IgM ELISA in parallel with the Euroimmun conventional Zika virus IgG ELISA (EI 2668-9601 G). Both Euroimmun assays use recombinant Zika virus nonstructural protein 1 as the antigen; the InBios Zika Virus Detect uses a recombinant Zika virus envelope glycoprotein as the positive antigen, an unspecified cross-reactive control, and reference cell antigens; and the Novatec and Abcam ELISAs use an unspecified Zika virus antigen.

Most tests evaluated provided algorithms that resulted in positive, negative, or equivocal results. However, the InBios kits account for antigenics in associated with secondary flavivirus infections and reports results as Zika virus positive, possible Zika virus positive, or presumptive other flavivirus positive or negative on the basis of calculations of optical density ratios obtained from a sample with the 3

\begin{tabular}{|c|c|c|c|c|c|c|c|c|c|}
\hline \multirow{2}{*}{$\begin{array}{l}\text { Sample } \\
\text { collection } \\
\text { dpo }\end{array}$} & \multicolumn{3}{|c|}{ In-house Zika virus diagnostic results } & \multirow[b]{2}{*}{$\begin{array}{c}\text { DENV } \\
\text { PRNT titer }\end{array}$} & \multicolumn{5}{|c|}{ Commercial Zika virus serologic assays results } \\
\hline & RT-PCR & $\begin{array}{l}\text { MAC- } \\
\text { ELISA }\end{array}$ & PRNT titer & & $\begin{array}{c}\text { Euroimmun } \\
\text { IgM }\end{array}$ & $\begin{array}{c}\text { Euroimmun } \\
\lg \mathrm{g}\end{array}$ & $\begin{array}{c}\text { Novatec } \\
\text { lgM }\end{array}$ & Abcam IgM & InBios IgM \\
\hline 12 & ND & Pos & $>40$ & Neg & Pos & Pos & Pos & Pos & Pos \\
\hline 9 & ND & Pos & $>40$ & Neg & Neg & Neg & Neg & Neg & Pos \\
\hline 4 & ND & Pos & $>40$ & Neg & Neg & Neg & Neg & Pos & Pos \\
\hline 27 & ND & Pos & $>40$ & Neg & Pos & Pos & Pos & Pos & Pos \\
\hline 39 & ND & Pos & $>40$ & Neg & Neg & Pos & Pos & Pos & Pos \\
\hline 11 & ND & Pos & $>40$ & Neg & Pos & Pos & Pos & Pos & Pos \\
\hline 109 & ND & Pos & 1,280 & 20 & Neg & Pos & Neg & Neg & Pos \\
\hline 49 & ND & Pos & $>40$ & Neg & Neg & Pos & Pos & Pos & Pos \\
\hline Unknown & ND & Pos & $>40$ & Neg & Neg & Neg & Neg & Neg & Pos \\
\hline 4 & ND & Pos & $>40$ & Neg & Pos & Neg & Pos & Pos & Pos \\
\hline 7 & ND & Pos & $>40$ & Neg & Neg & Pos & Neg & Neg & Pos \\
\hline 46 & ND & Pos & $>40$ & Neg & Neg & Pos & Pos & Pos & Pos \\
\hline Unknown & ND & Pos & $>40$ & Neg & Neg & Pos & Neg & Neg & Pos \\
\hline Unknown & ND & Pos & $>40$ & Neg & Neg & Pos & $\mathrm{Eq}$ & Pos & Pos \\
\hline 118 & ND & Pos & $>40$ & Neg & Neg & Pos & Neg & Neg & Pos \\
\hline 57 & ND & Pos & $>40$ & Neg & Pos & Neg & Pos & Pos & Pos \\
\hline 66 & ND & Pos & $>40$ & Neg & Neg & Pos & Neg & Eq & Pos \\
\hline 43 & ND & Pos & 40 & Neg & Neg & Pos & $\mathrm{Eq}$ & Pos & Pos \\
\hline 2 & ND & Pos & $>40$ & Neg & Neg & Neg & Pos & Neg & Pos \\
\hline 41 & ND & Pos & $>40$ & Neg & Pos & Pos & Neg & Neg & Pos \\
\hline 5 & ND & Pos & $>40$ & Neg & Neg & Neg & Neg & Neg & Pos \\
\hline 38 & ND & Pos & $>40$ & Neg & Neg & Pos & Neg & Neg & $\underline{\mathrm{PZ}}$ \\
\hline 4 & ND & Pos & $>80$ & Neg & Pos & Neg & Neg & Pos & Pos \\
\hline 6 & ND & Pos & $>80$ & Neg & Pos & Pos & Neg & Neg & Pos \\
\hline 2 & ND & Pos & $>80$ & Neg & Neg & Pos & Neg & $\mathrm{Eq}$ & Pos \\
\hline 12 & ND & Pos & 40 & Neg & Pos & Neg & Pos & Pos & Pos \\
\hline Unknown & ND & Pos & $>80$ & $\mathrm{Neg}$ & Pos & Neg & Pos & Pos & Pos \\
\hline 28 & ND & Pos & $>80$ & Neg & Pos & Pos & Neg & Neg & Pos \\
\hline 75 & ND & Pos & $>80$ & 20 & Neg & Pos & Neg & Neg & Pos \\
\hline 68 & ND & Pos & $>40$ & Neg & Neg & Pos & Neg & Neg & Pos \\
\hline 9 & ND & Pos & Neg & $>40$ & Neg & Neg & Neg & $\mathrm{Neg}$ & $\underline{\mathrm{PZ}}$ \\
\hline 7 & ND & Pos & Neg & $>40$ & $\mathrm{Neg}$ & Neg & Neg & $\mathrm{Neg}$ & $\underline{\mathrm{PZ}}$ \\
\hline 31 & ND & Pos & Neg & $>40$ & $\mathrm{Neg}$ & Neg & Neg & $\mathrm{Neg}$ & $\underline{P Z}$ \\
\hline 6 & ND & Pos & Neg & $>40$ & Pos & Pos & Neg & Neg & Pos \\
\hline 20 & ND & Pos & Neg & $>40$ & $\mathrm{Neg}$ & $\mathrm{Neg}$ & Pos & Pos & $\underline{\mathrm{PZ}}$ \\
\hline 36 & ND & Pos & Neg & $>80$ & Neg & Neg & Neg & Neg & $\mathrm{OF}$ \\
\hline Unknown & ND & Pos & 320 & $>5,120$ & Neg & Neg & Neg & Neg & Pos \\
\hline Unknown & ND & Pos & Neg & 40 & Neg & Neg & Pos & Neg & Neg \\
\hline 3 & ND & Pos & Neg & $>80$ & Neg & Neg & Eq & Neg & Pos \\
\hline Unknown & ND & Pos & Neg & $>640$ & $\mathrm{Neg}$ & $\mathrm{Neg}$ & Neg & Neg & Pos \\
\hline
\end{tabular}

*Bold indicates false-positive/false-negative results. Underlining indicates inconclusive results that required further testing. DENV, dengue virus; dpo, days postsymptom onset; Eq, equivalent; MAC-ELISA, IgM-capture ELISA; ND, not done; Neg, negative; OF, other flavivirus; Pos, positive; PRNT, plaque reduction neutralization test; PZ, possible Zika virus; RT-PCR, reverse transcription PCR. 
Table 3. Detection of IgM in RT-PCR-positive serum samples by using in-house and commercial Zika virus serologic assays*

\begin{tabular}{|c|c|c|c|c|c|c|c|c|c|}
\hline \multirow{3}{*}{$\begin{array}{l}\text { Sample } \\
\text { collection, dpo }\end{array}$} & \multirow{2}{*}{\multicolumn{3}{|c|}{ In-house Zika virus diagnostic results }} & \multirow{3}{*}{$\begin{array}{l}\text { DENV } \\
\text { PRNT }\end{array}$} & \multicolumn{5}{|c|}{ Commercial Zika virus serologic assays results } \\
\hline & & & & & \multirow{2}{*}{$\begin{array}{c}\text { Euroimmun } \\
\text { IgM }\end{array}$} & \multirow{2}{*}{$\begin{array}{l}\text { Euroimmun } \\
\text { IgG }\end{array}$} & \multirow{2}{*}{$\begin{array}{c}\text { Novatec } \\
\text { IgM }\end{array}$} & \multirow{2}{*}{$\begin{array}{l}\text { Abcam } \\
\text { IgM }\end{array}$} & \multirow{2}{*}{$\begin{array}{c}\text { InBios } \\
\text { IgM }\end{array}$} \\
\hline & RT-PCR & MAC-ELISA & PRNT & & & & & & \\
\hline 0 & Pos & Neg & ND & ND & Neg & Neg & Neg & Neg & Neg \\
\hline 2 & Pos & Neg & ND & ND & Neg & Neg & $\mathrm{Eq}$ & Neg & Pos \\
\hline 5 & Pos & Neg & ND & ND & Neg & Neg & Neg & Neg & Neg \\
\hline 7 & Pos & Pos & ND & ND & Pos & Pos & Pos & Pos & Pos \\
\hline 3 & Pos & $\mathrm{Neg}$ & ND & ND & $\mathrm{Neg}$ & $\mathrm{Neg}$ & Neg & Neg & $\mathrm{Neg}$ \\
\hline 2 & Pos & $\mathrm{Neg}$ & ND & ND & $\mathrm{Neg}$ & $\mathrm{Neg}$ & $\mathrm{Neg}$ & $\mathrm{Neg}$ & Pos \\
\hline 2 & Pos & Pos & ND & ND & $\mathrm{Neg}$ & $\mathrm{Neg}$ & Pos & $\mathrm{Neg}$ & Pos \\
\hline 3 & Pos & Neg & ND & ND & $\mathrm{Neg}$ & $\mathrm{Neg}$ & $\mathrm{Neg}$ & $\mathrm{Neg}$ & Pos \\
\hline 3 & Pos & $\mathrm{Neg}$ & ND & ND & $\mathrm{Neg}$ & $\mathrm{Neg}$ & Pos & $\mathrm{Neg}$ & $\mathrm{PZ}$ \\
\hline 2 & Pos & $\mathrm{Neg}$ & ND & ND & $\mathrm{Neg}$ & $\mathrm{Neg}$ & $\mathrm{Neg}$ & $\mathrm{Neg}$ & $\mathrm{Neg}$ \\
\hline
\end{tabular}

different antigens. Two independent laboratory technicians blindly evaluated the 5 assays by using the panel outlined, according to the manufacturer's instructions. Comparisons and performance calculations were conducted by the Quality Control Office of the National Microbiology Laboratory.

The assays generally showed reproducible results during independent evaluations, although specificity and sensitivity of each varied (Table 1). The Euroimmun IgM and IgG ELISAs and the Abcam IgM ELISA showed a specificity of $100 \%$ for negative specimens with similar results $(\geq 90 \%)$ for confirmed DENV-positive samples (Table 2, https://wwwnc. cdc.gov/EID/article/23/9/16-2043-T2.htm). The NovaTec ELISA showed a specificity of $66 \%$ for negative specimens and $70 \%$ for DENV-positive specimens. Although the InBios Zika Detect ELISA showed similar specificity results for flavivirus-seronegative specimens, it showed decreased specificity for DENV-positive samples. This assay incorrectly identified $40 \%$ of these samples as Zika virus IgM positive and $40 \%$ as possible Zika virus positive.

Although specificity is a key factor, for a front-line diagnostic test, sensitivity is a major factor in determining its usefulness. With appropriate diagnostic testing in place, including use of Zika virus conformational cross-PRNTs, false-positive results caused by specificity issues can usually be overcome. However, poor sensitivity will lead to false-negative results that might not be followed up by testing of additional sample collections. When compared with the in-house diagnostics (MAC-ELISA with PRNT confirmation), the IgM assays of Euroimmun, Abcam, and Novatec demonstrated sensitivities of $37 \%, 57 \%$, and $65 \%$, respectively. When we combined results of the Euroimmun IgM and IgG ELISAs, sensitivity increased to $82 \%$. The InBios Zika Virus Detect IgM assay correctly identified all confirmed Zika IgM-positive samples identified by the recommended diagnostic assays, resulting in a sensitivity of $100 \%$. The InBios ELISA also detected IgM in $50 \%$ of samples that were positive for Zika virus by RT-PCR, whereas the other assays did not detect IgM in most of these samples (Table 3).

\section{Conclusions}

The low sensitivity of most immunoassays evaluated could be improved by testing a repeat sample collected a few weeks after the initial specimen, although this sampling is not always practical, particularly if resources are limited. When performed in combination, the Euroimmun Zika Virus IgM and IgG ELISAs provide improved sensitivity. However, interpretation of recent versus past infections could be problematic, particularly when IgM results are negative and IgG results are positive. On the basis of our findings, the InBios Zika Virus Detect MAC-ELISA provides diagnostic results comparable to the CDC-based in-house MAC-ELISA for specimens collected from patients with primary flavivirus exposures (i.e., no detectable background immunity to DENV). A needed follow-up to our study will be further evaluation of IgM detection by commercial ELISAs involving cases of secondary flavivirus exposures or previous immunization to related viruses, such as yellow fever virus.

This study was supported by the Public Health Agency of Canada.

Dr. Safronetz is a research scientist in the Zoonotic Diseases and Special Pathogens Section, National Microbiology Laboratory, Public Health Agency of Canada, Winnipeg, Manitoba, Canada. His research interests include the ecology, epidemiology, and pathogenicity of emerging virus pathogens.

\section{References}

1. Dick GW, Kitchen SF, Haddow AJ. Zika virus. I. Isolations and serological specificity. Trans R Soc Trop Med Hyg. 1952;46: 509-20. http://dx.doi.org/10.1016/0035-9203(52)90042-4

2. Wikan N, Smith DR. Zika virus: history of a newly emerging arbovirus. Lancet Infect Dis. 2016;16:e119-26. http://dx.doi.org/10.1016/S1473-3099(16)30010-X

3. Weaver SC, Costa F, Garcia-Blanco MA, Ko AI, Ribeiro GS, Saade G, et al. Zika virus: history, emergence, biology, and prospects for control. Antiviral Res. 2016;130:69-80. http://dx.doi.org/10.1016/j.antiviral.2016.03.010

4. Duffy MR, Chen TH, Hancock WT, Powers AM, Kool JL, Lanciotti RS, et al. Zika virus outbreak on Yap Island, Federated States of Micronesia. N Engl J Med. 2009;360:2536-43. http://dx.doi.org/10.1056/NEJMoa0805715 
5. Lanciotti RS, Kosoy OL, Laven JJ, Velez JO, Lambert AJ, Johnson AJ, et al. Genetic and serologic properties of Zika virus associated with an epidemic, Yap State, Micronesia, 2007. Emerg Infect Dis. 2008;14:1232-9. http://dx.doi.org/10.3201/ eid1408.080287

6. Cao-Lormeau VM, Roche C, Teissier A, Robin E, Berry AL, Mallet HP, et al. Zika virus, French Polynesia, South Pacific, 2013. Emerg Infect Dis. 2014;20:1085-6. http://dx.doi.org/10.3201/ eid2011.141380

7. Campos GS, Bandeira AC, Sardi SI. Zika virus outbreak, Bahia, Brazil. Emerg Infect Dis. 2015;21:1885-6. http://dx.doi.org/10.3201/eid2110.150847
8. Grossi-Soyster EN, LaBeaud AD. Clinical aspects of Zika virus. Curr Opin Pediatr. 2017;29:102-6. http://dx.doi.org/10.1097/ MOP.0000000000000449

9. Landry ML, St George K. Laboratory diagnosis of Zika virus infection. Arch Pathol Lab Med. 2017;141:60-7. http://dx.doi.org/10.5858/arpa.2016-0406-SA

Address for correspondence: David Safronetz, Zoonotic Diseases and Special Pathogens, National Microbiology Laboratory, Public Health Agency of Canada, Winnipeg, MB, R3E 3R2, Canada; email: david.safronetz@phac-aspc.gc.ca

\section{October 2010: Zoonoses}

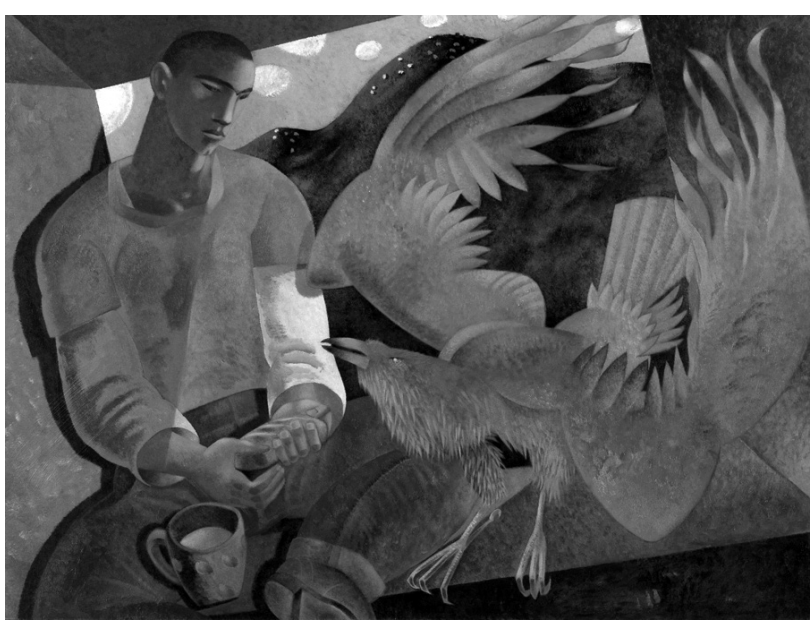

- Influenza A (H5N1) Viruses from Pigs, Indonesia

- Mobile Phone-based Infectious Disease Surveillance System, Sri Lanka

- Oral Fluid Testing during 10 Years of Rubella Elimination, England and Wales

- Human Monkeypox Outbreak Caused by Novel Virus, Sudan

- Therapeutic Drug Monitoring for Slow Response to Tuberculosis Treatment, Virginia

- Risk Factors for Pandemic (H1N1) 2009 Virus Seroconversion among Hospital Staff, Singapore

- Personal Protective Equipment and Oseltamivir Prophylaxis during Avian Influenza A (H7N7) Epidemic, the Netherlands

- Bloodstream Infections among HIV-Infected Outpatients, Southeast Asia

- Changing Epidemiology of Pulmonary Nontuberculous Mycobacteria Infections

- Mortality Patterns for Hemorrhagic Fever with Renal Syndrome Caused by Puumala Virus
- Pandemic (H1N1) 2009 Virus on Commercial Swine Farm, Thailand

- Toxoplasma gondii Oocyst-specific Antibodies and Source of Infection

- Predicting Need for Hospitalization of Patients with Pandemic (H1N1) 2009

- Imported Lassa Fever, Pennsylvania

- Type 2 Diabetes Mellitus and Increased Risk for Malaria Infection

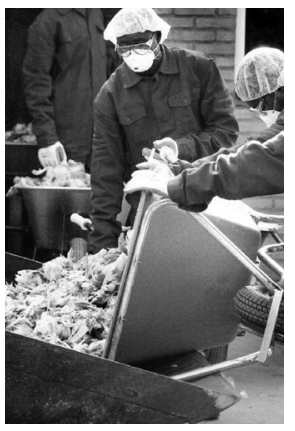

- Human Parvovirus 4 Infection in Sub-Saharan Africa

- Artesunate Misuse and Malaria in Traveler Returning from Africa

- Severe Plasmodium vivax Malaria, Brazilian Amazon

- Erythema Migrans-like Illness among Caribbean Islanders

- Pandemic (H1N1) 2009 and Seasonal Influenza A (H1N1) Co-infection, New Zealand

- Chemokine Receptor $5 \Delta 32$ Allele and Severe Pandemic (H1N1) 2009

- Klassevirus Infection in Children, South Korea

- Human Cases of MRSA CC398 Infection, Finland

- Hepatitis E Virus Genotype Diversity in Eastern China

- Emergence of Oseltamivir-Resistant Pandemic (H1N1) 2009 Virus within 48 Hours

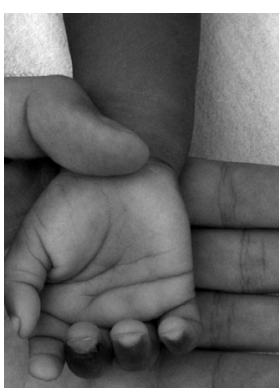

- Avian Leukosis Virus Subgroup J in Layer Chickens, China

- Healthcare Worker Acceptance of Pandemic (H1N1) 2009 Vaccination, Morocco

- New Rural Focus of Plague, Algeria

- Superbug: The Fatal Menace of MRSA

\section{EMERCING}

INFECTIOUS DISEASES https://wwwnc.colc.gov/eid/articles/issue/16/10/table-of-contents 